\title{
SURVIVABILITY AND QUALITY OF SERVICE IN MOBILE WIRELESS NETWORKING ${ }^{1}$
}

\author{
Stuart Milner, Sohil Thakkar, Karthikeyan Chandrashekar, Wei-Lun Chen \\ Institute for Systems Research and A.J. Clark School of Engineering \\ University of Maryland at College Park
}

\begin{abstract}
This paper focuses on two aspects of survivable, fault tolerant, mobile wireless networking: 1) dynamic IP affiliation and connectivity amidst router (base station) failures; and 2) quality of service based-mobility and routing. In both cases, we discuss the protocols developed, and we evaluate their performance using discrete event simulation. The processes and protocols developed represent enhancements to our previous work in basestation oriented tactical networks. We also discuss implications for scalability.
\end{abstract}

\section{BACKGROUND}

Our research has focused on the scalability of wireless networks based on dynamic base-stations and hosts [1] as well as their associated routing, mobility, and quality of service (QoS) models [2,3,4]. In this paper, we focus on the design, development and evaluation of: 1) protocols for dynamic IP affiliation of hosts and their connectivity amidst router (base station) failures (i.e., survivability); and 2) processes for routing and mobility based on QoS.

The survivability and QoS models are enhancements to our routing and mobility models presented in $[2,4]$, in which both routers and hosts can be mobile. Our approach to dynamic IP affiliation (handoff) allows hosts to make seamless handoff decisions based on router advertisements while mobile. This differs from Mobile IP, in which handovers are accomplished by rerouting. In addition, Mobile IP does not specify a "handoff" protocol under continuous mobility [5].

Host handoff is achieved in a proactive manner with the IP address maintained by the host and the network layer while the host affiliation with a new router is handled at the MAC layer [2]. The host address databases are updated at the MAC layer and at the network layer. Previous work has demonstrated the scalability of this approach [2] and its operational potential [3].

The mobility protocol described in [2] ensures data connectivity when the hosts and routers are mobile. In this paper we describe the new survivability or fault tolerant protocol that provides host connectivity in the face of host or router node catastrophic failures in tactical situations.
Of particular concern is router failure. It is essential to discover router failure in a timely fashion and to identify another router to assume the responsibility of the failed router node.

The survivability protocol ensures data connectivity by:

- Discovering router failures and identifying replacements

- Ensuring hosts of the failed router re-align with the newly identified router

- Modifying the IP data forwarding mechanism so that the re-aligned hosts remain connected to the network

Because hosts may move out range of their "home router", their mobility must be managed to prevent them from becoming orphaned. Our enhanced approach to this uses a mobility protocol that allows hosts to make proactive decisions that allow binding to a "preferred" router based on QoS variables (e.g., router congestion) and MAC layer broadcasts.

\section{Scalability of Wireless Networks}

A major challenge for operational use of mobile, wireless networks is to empirically determine their scalability - the effects of network size (thousands of dynamic hosts/links/routers) on performance (e.g., latency, signaling, overhead) and comnunications complexity (i.e., control messages). This requires a full-scale model of the mobile, wireless network (five layers of the Internet protocol suite).

In the largest such scalability effort known to us, we used discrete event simulation (OPNET) to model over 2000 fully mobile, wireless nodes: routers (50), hosts (2000) and application servers (25). We made some crucial observations from unified, systems-oriented, large-scale simulation experiments. One of the most important was that of routing overhead associated with wireless Open Shortest Path First (OSPF) remained constant, regardless of the amount of application traffic. Furthermore, for a wide range of application traffic load, OSPF traffic and relative packet loss rate remained relatively the same. We concluded that: 1) the mobility protocol overhead was minimal; 2) binding load increased in a linear manner as the number of hosts initiating binding increased; 3) the

\footnotetext{
1 This work was supported by the Defense Advanced Research Projects Agency.
} 
communication complexity between routers did not exceed that of wire line complexity; and 4) increasing areas or subnets facilitates scalability and OSPF overhead remains the same regardless of network traffic. Finally, we concluded that the dynamic IP, a cellular-like mobility protocol, and hierarchical base-station-oriented network architecture are the key factors contributing to scalability.

Prior to these simulations, a full-scale prototype system using this base-station architecture was developed and implemented in real experiments, thereby providing a proof-of-concept for the Department of Defense [3]. The simulations in [2] verified the scalability of this approach for DoD.

Since the current survivable and QoS models are enhancements to the scalable models in [2], we do not require the large-scale simulations previously conducted. Instead, we focus primarily on the performance of the current models and suggest implications and generalizations regarding their scalability.

\section{Simulation and Modeling Methodology}

The modeling and simulation is based on OPNET versions 7 and 8 , which we enhanced to accommodate our mobility and routing models $[2,3,4]$. In fact, some of our enhancements have been incorporated into the latest versions of OPNET. To maximize the number of nodes and to reach our goal of over 2000 nodes as well as for purposes of performance scalability and simulation efficiency, we optimized memory management by profiling the simulations and eliminating "packet leaks". The IEEE 802.11 standard is used for both the router backbones and the mobile host LANs. Each router interface as well as the affiliated hosts forms a Basic Service Set (BSS). A group of these sets forms an Extended Service Set (ESS). Thus, a hierarchical model is possible by localizing routing and mobility information within the ESS areas. We use a modified version of the wireless OSPF protocol to implement "areas" or subnets.

\section{SURVIVABILITY MODEL}

The survivability or fault tolerant protocol is based on the point-to-multipoint OSPF, mobility and IP models developed in $[2,3]$. To achieve survivability once a router fails, we implemented a simple distributed election scheme for choosing a replacement router that adds no communication complexity. Modifications were made to the mobility protocol $[2,3]$ to enable the hosts to identify their "home agent" router failures and re-align to the new active router. Finally modifications to the IP forwarding process ensure data connectivity for re-aligned hosts.

\section{Fault Tolerant Routing Protocol}

The fault tolerant routing includes: 1) determination of a router failure; 2) re-assignment of the failed router's functions to a new active router; and 3) dissemination of this information to all other routers in the network. Once this is achieved, the IP forwarding table on all the active routers is updated so that packets are forwarded correctly. Also, remote and/or locally bound hosts associated with the failed router must acquire a new "home agent" and, subsequently, bind to it.

In our approach, OSPF is used to discover a router failure. By definition, when all the router interfaces are disconnected from the network, HELLO messages cannot be received and that router is declared inoperative. The other routers that had been connected to the failed router will no longer include it in the link state announcements they generate [6]. When the new route table is computed, there will no longer be entries for the failed router. In addition, when this failure is discovered, each router executes an algorithm, which determines which of the active routers will take over the functionality of the failed router.

In order to determine a scalable, low overhead, means to select the new router, we implemented a simple and novel approach. We developed an algorithm that uses the OSPFassigned ID and a decision rule that determines the reassignment of functionality from the failed router to a new active router, which has the lowest ID of the remaining active routers. The advantage is that no explicit message exchange is needed to determine the replacement router as every router inherently has the necessary information.

Specifically:

Set $B(t)=\{$ All active routing nodes in a network at time $t\} t$ $\in \mathrm{R}^{+}$

Set $C(n)(t)=\{$ All active nodes that are "take over" router for $\mathrm{n}$ failed nodes at time $\mathrm{t}\}, \mathrm{n}=0,1,2,3 \ldots$.

Hence $\mathrm{C}(0)(0)=\mathrm{B}(0)$.

For any time $t$,

$B(t)=\cup C(n)(t) n=0,1,2,3 \ldots$ (i. e. $C(0)(t) \cup C(1)(t) \cup$ $\mathrm{C}(2)(\mathrm{t}) . .$.

Rule : At any time $t$, if a node $\mathrm{N}$ fails

Select lowest ID router $T \in C(m)(t)$ such that $m$ satisfies following:

Let $f(n)=|C(n)(t)|$

$m=\underset{n=0.1 \ldots}{\operatorname{argmin}} f(n) \& f(n)>0$

where $||=$. Number of elements in a set

Router $\mathrm{T}$ takes over the functionality of the failed router $\mathrm{N}$, and all the other failed router nodes for which $\mathrm{N}$ had previously assumed their functionality. Since all active routers use this rule, they know about the replacement router as soon as they learn about the failed router. A table entry is created in a list, which is indexed by failed node and points to the newly identified replacement router. The IP process includes this list, and if a packet is addressed to the failed router, it is mapped to the new active 
replacement router and forwarded accordingly. Thus, the replacement router finally gets the packet for host belonging to failed router. Data forwarding from the replacement router to the host is as specified in the mobility protocol in [1].

\section{Fault Tolerant Mobility Protocol}

To ensure connectivity, a failed router's remotely bound hosts need to learn about their Home Agent's failure. This is because remotely bound hosts are dependent on the Home Agent for receipt of data. The failed router's local hosts, upon failing to receive mobility advertisements, will locally bind to a new router and initiate a new remote binding with it [1].

Each Home Agent periodically advertises a remote bind HELLO message to each remotely bound host. A host that doesn't receive this advertisement concludes that the Home Agent has failed.

Upon Home Agent failure, hosts remotely bound to it will fail to receive the bind HELLO, i.e., those hosts currently affiliated to a router other than their Home Agents. The host then initiates a remote binding message to the same Home Agent. The purpose of a remote binding is to inform the new Home Agent, the care of address of the Foreign Agent to which the host is currently bound. The new Home Agent can then forward all messages addressed to this host to the Foreign Agent.

In our approach if a Home Agent becomes inoperable, another router assumes the functionality of that failed Agent; however, the new Home Agent does not know the "Care of Address" of the Foreign Agent to which a host (belonging to the failed router) is affiliated.

The new Home Agent then receives the remote binding message sent by the host, due to the IP forwarding modification. Hence the new agent learns of the care of address of the remotely bound host.

In the case of the host not receiving a HELLO message while the original Home Agent is still active, the remote binding message sent by the host just serves as an updated remote binding and doesn't adversely affect the protocol except for the transmission of a redundant message. Thus, the protocol ensures that the hosts remain connected to network irrespective of their location at the time of their Home Agent failure.

\section{QUALITY OF SERVICE MODEL}

\section{OoS-Based Handoff: Design and Protocol}

The QoS-based handoff procedure enables the host to evaluate its binding to a router and, if necessary, bind to another router based on signal strength comparisons and advertised preference value [2]. The model uses a preference value to reflect the status of the router, and it is expected that a host will choose a router that provides the best service defined by that metric. Several preference value metrics (e.g., IP queuing delay, processing rate) were evaluated, and after their functional equivalence was determined, IP queuing delay was chosen. Upon deciding to perform a new local binding [2] based on these factors, a host will send a bind message to the router that advertises the best IP queuing delay metric, and a QoSbased handoff is achieved.

Since the host decision to bind is based on signal strength and a deterministic QoS preference value, it is possible that a host may exhibit "Ping-Pong" behavior - host oscillation between routers due to IP queuing delay variation induced by the host movement. The hosts first bind to a router with a more favorable delay. That movement could result in an increase in IP queuing delay of the new router and a corresponding decrease in the previous router delay, and so on. This can cause oscillatory host binding behavior, which leads to degraded network performance due to repeated initiations of the local binding process. This Ping-Pong effect is prominent during "Group Binding" i.e., when a group of hosts change their binding simultaneously.

To negate the Ping-Pong effect, preference values were assigned based on the range of the IP queuing delay. Table 1 shows the preference values we used as a function of IP queuing delays. An increment of the preference value corresponds to an order of magnitude improvement in the queuing delay.

\begin{tabular}{|c|c|c|}
\hline $\begin{array}{c}\text { Interface } \\
\text { Cost }\end{array}$ & $\begin{array}{c}\text { Preference } \\
\text { Value }\end{array}$ & $\begin{array}{c}\text { Average IP } \\
\text { Queuing Delay }\end{array}$ \\
\hline 1 & 6 & $<0.0001(\mathrm{sec})$ \\
\hline \multirow{2}{*}{2} & 5 & $<0.001(\mathrm{sec})$ \\
\cline { 2 - 3 } & 4 & $<0.01(\mathrm{sec})$ \\
\hline \multirow{2}{*}{3} & 3 & $<0.1(\mathrm{sec})$ \\
\cline { 2 - 3 } & 2 & $<1(\mathrm{sec})$ \\
\hline 4 & 1 & $\geq 1(\mathrm{sec})$ \\
\hline
\end{tabular}

\section{Table 1. Interface Costs and Preference Values}

Each router updates its preference value based on the average IP queuing delay for the last 10 samples. This ensures that even if a number of hosts bind to the new router the preference value would still be stable, and therefore, the hosts will not oscillate between routers. The hosts are thus able to maintain stable affiliations and achieve a distinct improvement in service.

Host performance is further improved by providing a multi-criterion rebind trigger for the hosts. In this scheme a host will rebind if the signal strength from its router falls below the threshold or if the QoS metric advertised by its router falls below the threshold. This adds another degree of freedom to the host in making the binding decision, i.e., the host can make intelligent handoffs based on whether it requires improved connectivity or improved service. We found that the QoS-based handoff will provide the best first hop connection. This improves the service the host 
gets from its router. However to ensure an end-to-end improvement in service for the host, the routing mechanism should also incorporate the preference value metric.

\section{OoS-Based Routing}

The handoff algorithm is useful in selecting the most capable, first hop router but does not guarantee better endto-end service. This is because the hop-count for one packet transfer is unpredictable and may be routed subsequently to a highly congested router.

To ensure end-to-end QoS for any source-destination pair, we need a dynamic routing scheme that utilizes the QoS metric (IP queuing delay) to generate routes. Also the routes should be updated dynamically as the QoS metrics in the routers change.

We modified the OSPF to support QoS-based link costs. Link State Updates (LSUs) are generated when connectivity and QoS metrics change. To prevent frequent initiations of LSUs due to subtle variations in IP queuing delay, the delay is grouped into ranges and interface costs are assigned based on these ranges in Table 1. A moving average is computed for queuing delay, and this interface cost is advertised. The Dijkstra algorithm will use these costs to determine the shortest path for each sourcedestination pair. Since the routing is dynamic, the paths are continuously updated based on the QoS metric.

Finally, use of both the handoff and QoS routing models was used in our simulations to ensure the best quality of service effort in the network.

\section{RESULTS}

\section{Fault Tolerant Model}

As mentioned previously, when a router failure is discovered, a new router is selected, and packets can be sent and received by remotely bound hosts. However, packets are lost during the interval between the home agent failure and the time when a replacement router assumes the Home Agent responsibility. The host reconnect latency and packet loss during that time depends on both the HELLO message interval and the route computation.

We conducted experiments to determine the effects of OSPF overhead with respect to host reconnect latency and packet latency. The simulation consisted of 10 routers, a host and a server. The host's Home Agent router was terminated during the simulation. We measured the reconnect time and corresponding OSPF overhead for different values of OSPF HELLO interval.

Figure 1 shows the relationship between reconnect time and OSPF overhead head as a function of OSPF HELLO interval. We found an increase in latency and packet loss as the HELLO interval increases. As expected, decreasing the HELLO interval causes the network to react faster, thereby reducing reconnect time and packet loss, but increasing network overhead. For a HELLO interval set to 5 seconds, reconnect time is 34 second as compared to 101 seconds when the HELLO interval is set to 20 seconds. However, the average OSPF overhead reduces from 5925 $\mathrm{bits} / \mathrm{sec}$ to $4309 \mathrm{bits} / \mathrm{sec}$ when HELLO interval is changed from 5 seconds to 20 seconds. We conclude that the smaller the OSPF HELLO interval, the higher the network overhead and the faster the reconnect response. Similar results were obtained for packet loss.

Since no additional messages were exchanged in the case of OSPF, and one additional packet was added to the mobility protocol, we can generalize from our previous work [2] that the communication complexity is negligible, and, by implication, the fault tolerant protocol is scalable.

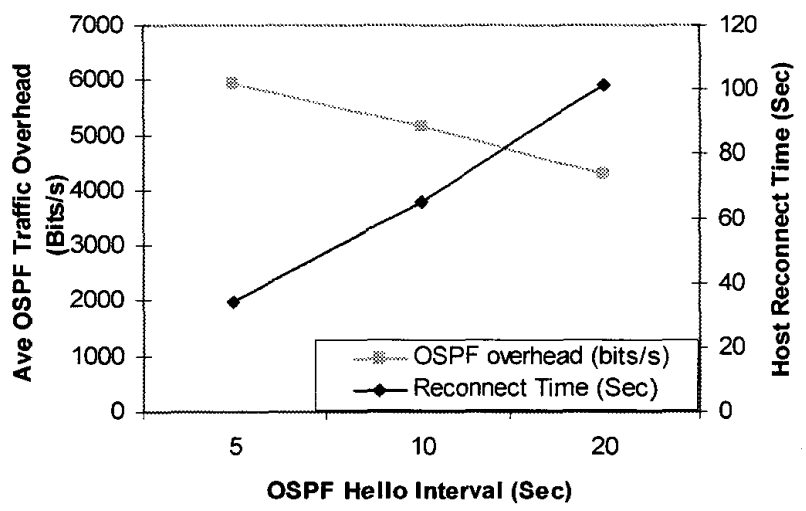

\section{Figure 1. Relationship between OSPF Overhead, Reconnect Time and HELLO Interval}

Although the host reconnect (remote binding) time varies on the order of tens of seconds and hosts do not receive packets during that interval, those hosts can start sending packets as soon as they locally bind to the foreign agent router. Table 2 shows the time for a new local binding following home agent failure for numbers of hosts, which have lost their home agent. This new local binding lasts only a few seconds and depends on the router advertisement interval [2]. The variability is generally small. However, the larger variability in the case with 15 hosts is due to one host's anomalous behavior.

\begin{tabular}{|l|l|l|l|l|l|}
\hline \# Hosts & $\mathbf{1}$ & $\mathbf{5}$ & $\mathbf{1 0}$ & $\mathbf{1 5}$ & $\mathbf{2 0}$ \\
\hline Mean & 1.99 & 2.10 & 2.17 & 3.50 & 3.66 \\
\hline Std.Deviation & 0 & .04 & .16 & 2.39 & .55 \\
\hline
\end{tabular}

Table 2. New Local Binding Latency (seconds)

Router failure is discovered when the routing table is recalculated (e.g., every 60 seconds). If a router fails, packets cannot be received by its bound hosts until they make a new remote binding is made to the 
replacement router. That interval, during which packets are lost, depends on the timely receipt of LSUs for route table convergence. We observed that this interval can last as much as 90 seconds or as little as 35 seconds (for a HELLO interval of $10 \mathrm{sec}$ ); and, the closer the LSUs are received to the OSPF route computation time, the greater the likelihood that the new routing tables will be computed at the next scheduled cycle. In other words, if information about a router failure were not propagated in a timely manner, recovery would occur in the next route computation interval ( 60 seconds).

\section{Quality of Service}

To investigate the effectiveness of the QoS handoff algorithm, a simulation was run with 50 mobile hosts and 10 fixed routers for a period of 600 seconds. One half of these hosts were configured to use the signal strength based binding algorithm [1] to initiate hand-offs and the other half used the QoS-based model. Half the routers were configured with heavy background traffic (approximately $5 \mathrm{~K}$ packets/second per router) to create congestion. Each host was configured to generate FTP requests at random times, using an exponential distribution with a mean request time of 30 seconds.

Since the signal strength from congested routers was stronger than the less congested routers, we ensured that hosts using the signal strength only model for hand-offs would always bind to those congested routers and thereby would serve as a benchmark.

The FTP response time for hosts using the QoS handoff and the signal strength only approaches are given in Figures 2 and 3 respectively. Response times are plotted on per request bases at one-second intervals. The response time for hosts using the QoS scheme ranges from .2 to .6 seconds; whereas, in the signal strength only based scheme, the range is between 2 and 4 seconds. The exponential increase in response time in Figure 3 resulted from an excess of traffic at the router. Note that the increased router traffic was subject to random background congestion traffic.

In the QoS scheme, the hosts always bind to the lesscongested routers and hence do not incur delays due to lost packet retransmissions. However in the signal-strength scheme the hosts will always bind to the congested routers and hence incur substantial congestion caused by retransmission delays. If this network were to be configured for UDP traffic, the congestion would result in even higher packet loss. Thus, the QoS handoff scheme combined with the QoS routing ensures higher packet delivery rate and reduced delay.

Although this combined approach improves packet delivery and reduces delay, the routing overhead is increased due to repeated route regeneration whenever the QoS metric changes significantly.

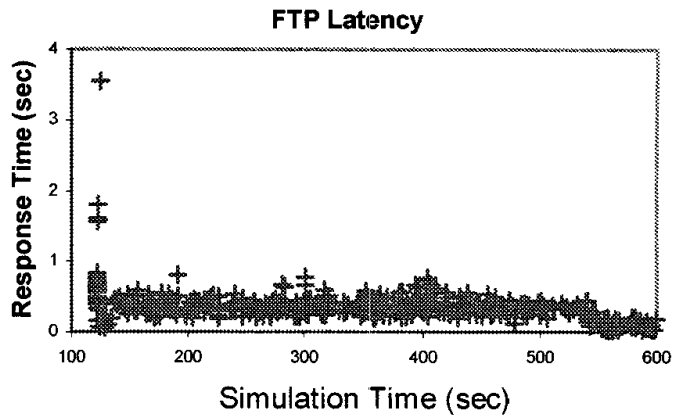

Figure 2. QoS Model

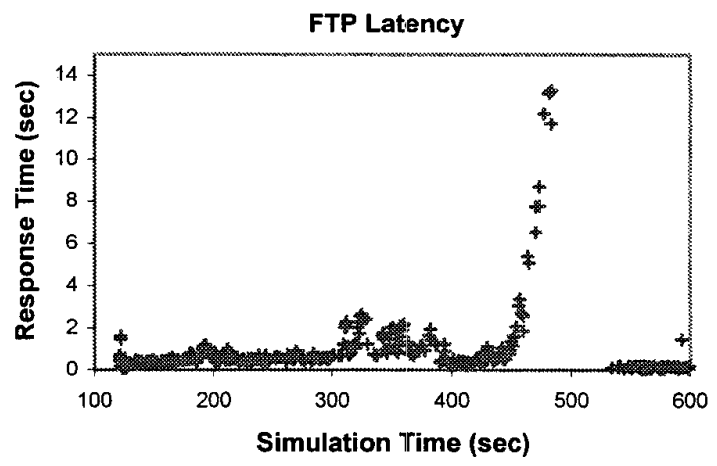

Figure 3. Signal Strength Only Model

To determine the extent of QoS routing overhead, the scenario described in the first paragraph of this section was used but with all hosts configured to use the QoS handoff scheme. Two such scenarios were run, one using the QoS routing and the other using routing (OSPF) without QoS (Figure 4).

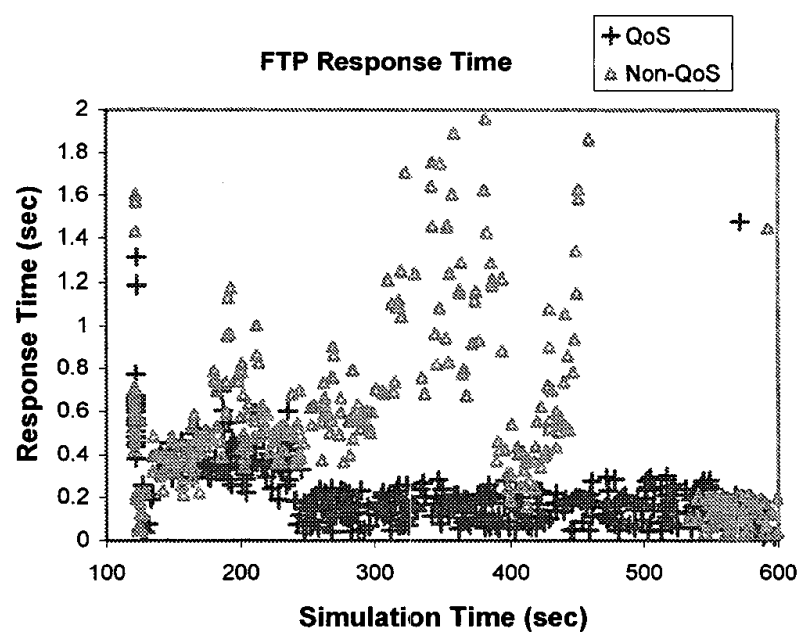

Figure 4. QoS and Non-QoS Routing 
In general, improved FTP response time occurs when QoS routing is used. Also, there is greater response time and considerably more variability when the non-QoS routing approach (signal strength only) is used.

On the other hand, to compare routing overhead (OSPF messages) for both the QoS-based and non-QoS schemes (Figure 5), simulations were conducted using the same scenario above. The non-QoS scheme (using OSPF only) generates minimal overhead and is relatively constant since only HELLO messages were sent.

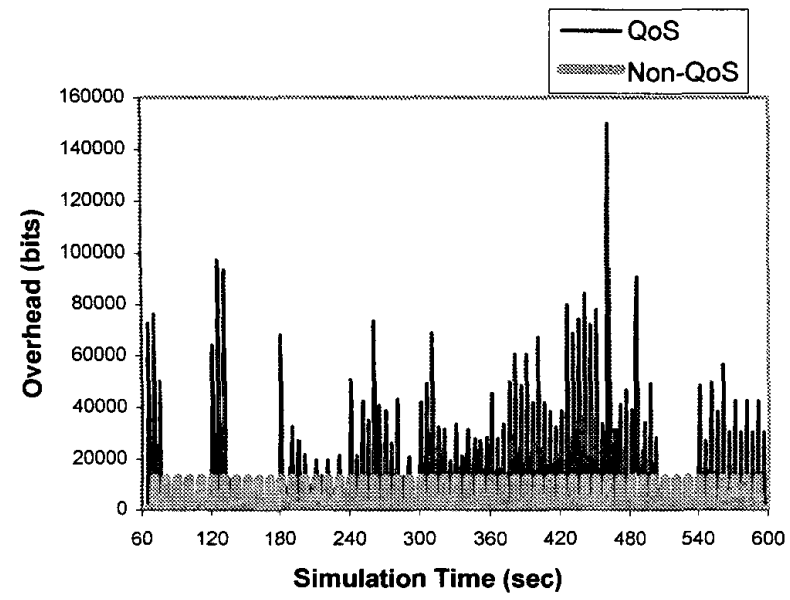

Figure 5. Routing Overhead

The QoS overhead is substantially greater than the nonQoS approach. It peaks at approximately $150 \mathrm{~Kb}$ as compared to $12 \mathrm{~Kb}$ for non-QoS approach. This is because the IP queuing variation results in cost changes, thereby initiating link state update exchange and route regeneration. Frequent changes in costs can result in unstable routes resulting in degraded host performance.

To limit the problem of routing computational and communications complexity, the frequency of LSUs generated due to the QoS metric change must be controlled. This is achieved by monitoring the metric at periodic intervals. In this experiment the interval was set to 1 minute, which is also the OSPF route computation interval.

\section{CONCLUSIONS}

We have designed, developed and evaluated models for two aspects of mobile wireless networking: 1) dynamic IP affiliation and connectivity amidst router (base station) failures; and 2) quality of service based-mobility (hosts/end users) as well as quality of service-based routing. In both cases, we discussed their detailed models as well as their performance and scalability using OPNET for discrete event simulation.

The survivable model uses OSPF routing processes to determine router node failures. Since no additional messages were exchanged in the case of OSPF, and one additional packet was added to the mobility protocol, we can generalize from our previous work [2] that the communication complexity is negligible, and, by implication, the fault tolerant protocol is scalable.

The host reconnect latency depends on the OSPF HELLO and route computation intervals. We found a tradeoff between OSPF overhead and host reconnect time as a function of the HELLO interval - as that interval increases, overhead decreases and host reconnect time increases.

An enhanced handoff decision model based on using router state information as well as signal strength measurement improves QoS or performance. Using the QoS routing algorithm improves end-to-end performance but increases overhead. More research is needed to determine the relationship between the QoS, frequency of routing change, and performance.

\section{REFERENCES}

1. S. D. Milner, S. L. Bernstein, C. W. Niessen, and D. T. White, Wireless Warfighter's Internet, Proc. MILCOM'97, November 1997.

2. S. D. Milner, J. E. Wieselthier, R. R. Iyer, K. Chandrashekar, S. Thakkar, and G. D. Nguyen. Scalability Of Dynamic Wireless Tactical Networks, Proc. MILCOM 2001.

3. Final Report for On Board Switch, BBN Technologies and TRW Tactical Systems, prepared for DARPA and AFRL, 22 January 2000.

4. S. D. Milner, K. Chandrashekar, S. Thakkar, and C-H Chen . Routing and Mobility Performance in Wireless Base-Station Networks, Proc. OPNETWORK 2001.

5. C.E. Perkins. Mobile IP, IEEE Communications Magazine, May 2002.

6. OSPF Version 2. RFC 2178, July 1997, IETF.

7. OPNET. Www.opnet.com. 